\title{
Progressive Degeneration and Inhibition of Peripheral Nerve Regeneration in the SOD1-G93A Mouse Model of Amyotrophic Lateral Sclerosis
}

\author{
Binbin Deng ${ }^{a}$ Wenjing Lva Weisong Duan ${ }^{a}, b, c$ Yakun Liu, ${ }^{a, b, c}$ Zhongyao Li $i^{a, b, c}$ \\ Yanqin Ma ${ }^{d}$ Guisen Zhang ${ }^{d}$ Xueqin Songa,b,c Can Cui ${ }^{a} \quad$ Xiaoming Qj ${ }^{a}$ Yuan Li $^{a}$ \\ Chunyan Lia,b,c
}

aDepartment of Neurology, Second Hospital of Hebei Medical University, Shijiazhuang, 'Institute of Cardiocerebrovascular Disease, Shijiazhuang, 'Neurological Laboratory of Hebei Province, Shijiazhuang, dJiangsu Nhwa Pharm. Co.Ltd, Nantong, P.R.China

\section{Key Words}

Peripheral nervous system • SOD1-G93A • Myelination • Degeneration • Regeneration

\begin{abstract}
Background: Myelination, degeneration and regeneration are implicated in crucial responses to injury in the peripheral nervous system. Considering the progression of amyotrophic lateral sclerosis (ALS), we used the superoxide dismutase 1 (SOD1)-G93A transgenic mouse model of ALS to investigate the effects of mutant SOD1 on the peripheral nerves. Methods: Changes in peripheral nerve morphology were analyzed in SOD1 mutant mice at various stages of the disease by toluidine blue staining and electron microscopy (EM). Schwann cell proliferation and recruitment of inflammatory factors were detected by immunofluorescence staining and quantitative reverse transcription PCR and were compared between SOD1 mutant mice and control mice. Furthermore, western blotting (WB) and TUNEL staining were used to investigate axonal damage and Schwann cell survival in the sciatic nerves of mice in both groups. Results: An analysis of the peripheral nervous system in SOD1-G93A mice revealed the following novel features: (i) Schwann cells and axons in mutant mice underwent changes that were similar to those seen in the control mice during the early development of peripheral nerves. (ii) The peripheral nerves of SOD1-G93A mice developed progressive neuropathy, which presented as defects in axons and myelin, leading to difficulty in walking and reduced locomotor capacity at a late stage of the disease. (iii) Macrophages were recruited and accumulated, and nerve injury and a deficit in the blood-nerve barrier were observed. (iv) Proliferation and the inflammatory micro-environment were inhibited, which impaired the regeneration and remyelination of axons after crush injury in the SOD1-G93A mice. Conclusions: The mutant human SOD1 protein induced axonal and myelin degeneration during the progression of ALS and participated in axon remyelination and regeneration in response to injury.

\begin{tabular}{ll}
\hline Chunyan Li & Department of Neurology, Second Hospital of Hebei Medical University \\
& Shijiazhuang 050000, Hebei (P.R. China) \\
& E-Mail hebeichunyanli@aliyun.com
\end{tabular}
\end{abstract}

\section{KARGER}




\section{Introduction}

Amyotrophic lateral sclerosis (ALS) is a fatal neurodegenerative disease characterized by muscle weakness and progressive paralysis. Superoxide dismutase 1 (SOD1) is a ubiquitous cytosolic enzyme; mutations in the $\mathrm{Cu} / \mathrm{Zn}$ Sod1 gene are a common cause of familial cases of this disease [1,2]. Transgenic mice expressing the mutated human Sod1 gene are used as a classical model of ALS, in terms of both the mechanism and treatment [3, 4]. The mutant gene shows a toxic gain of function phenotype, which leads to paralysis and death from respiratory failure in the animal model [5].

The progression of motor neuron disease in patients and animal models of ALS has been well characterized in terms of neuropathology and behavioral motor deficits [5]. Recent studies have shown motor neuron death, and microglia and astrocytes are activated during disease progression in the CNS $[6,7]$. However, alterations in the peripheral nervous system (PNS) are rarely mentioned.

The PNS originates from the outer layer of the neural crest. After birth, immature Schwann cells migrate and envelop large-diameter axons to establish a 1:1 relationship [8, 9]. A number of factors that affect peripheral nerve development are involved in this process, including transcription factors [10], microRNAs [11], laminin [12] and signaling molecules [13]. Mutations in the hSod1 gene in mice and humans result in muscular atrophy and peripheral nerve defects. For this reason, we determined whether a mutation in the hSod1 gene affected the development of the peripheral nerves and influenced their normal physiological function.

Peripheral nerves communicate between neurons and muscles [14, 15]. Degeneration of motor axons in the PNS is a crucial and significant pathological feature in patients and in animal models of ALS, and it is linked to defects in axonal transport and message transmission [16]. At a late stage, the expression of the SOD1-G93A mutant coincides with the occurrence of axon degeneration and behavioral deficits that are linked to the peripheral nerves. The PNS undergoes a period of cell death in subjects with ALS $[17,18]$. We analyzed changes in the morphology of the sciatic nerve in SOD1 mice at different times. Inflammation is a prominent pathological feature in patients and the animal model, along with peripheral nerve injury that is characterized by macrophage recruitment and activation. An interesting question is whether myeloid macrophages that are linked to the clearance of myelin debris in the PNS participate in ALS[19]. We evaluated the timing of severe axonal degeneration and inflammatory responses in the PNS.

Wallerian degeneration, a process in which the axon and myelin degenerate, is a cellular response activated by the injured axon accompanied by dedifferentiation of Schwann cells (SC)[20, 21]. Immune cells are recruited to the injured nerve to remove myelin fragments and promote axon regeneration and SC remyelination to restore motor function [19]. Mutant human SOD1, which shows a toxic gain of function phenotype, leads to abnormal changes in neurons and glia. However, successful axon regeneration relies on the coordinated responses of axons and glia. Therefore, we observed the alterations in Wallerian degeneration in the SOD1-G93A mice; macrophages were examined and SC proliferation was detected. The mutant human SOD1 affected the formation of the inflammatory environment and axon regeneration.

\section{Materials and Methods}

\section{Mice}

SOD1 transgenic mice (B6SJL-TgN [SOD1-G93A] 1 Gur) were purchased from Jackson Laboratory. PCR was performed on tail DNA samples to confirm the expression of the mutant gene in the offspring. Mice designated for experiments were bred under controlled conditions: a 12-h light/dark cycle, $60 \pm 10 \%$ relative humidity, and $22 \pm 1^{\circ} \mathrm{C}$.

\section{KARGER}




\section{Antibodies and Chemicals}

The following antibodies and other reagents were used: NF160 (Abcam, ab7794), NF-H (Abcam, ab8135), MBP (Abcam, ab7349), CD68 (Abcam, ab76308), MCP-1 (Abcam, ab8101), CD86 (Abcam, ab213044), TNF- $\beta$ (Proteintech, 18978-1-AP), S100 (Abcam, ab14849), BrdU (Abcam, ab1893), CHAT (Abcam, ab178850), NeuN (Millipore, ABN78), $\beta$-actin (Proteintech, 60008-1), Hoechst stain (1:100, Invitrogen), secondary antibodies for western blotting (Rockland Immunochemicals, USA), fluorescein isothiocyanate secondary antibodies (Jackson ImmunoResearch, West Grove, PA), TUNEL apoptosis assay kit (Beyotime Institute of Biotechnology, C1089), RNeasy Lipid Tissue Mini Kit (QIAGEN, 74804).

\section{Animal Surgery}

Surgery was performed on mice using previously described methods [22, 23]. Thirty-day-old mice were anesthetized with $2 \%$ pentobarbital sodium. The sciatic nerve was exposed after a skin incision and muscle dissection. The sciatic nerve was crushed with forceps for $30 \mathrm{~s}$ at the mid-thigh level of the nerve, at the site located $45 \mathrm{~mm}$ from the tip of the third toe. Mice received appropriate care after the sutures were placed at the end of the surgery.

\section{Western Blot Analysis}

Tissues were harvested from the sacrificed mice and transferred to tubes. Proteins were extracted using a protein extraction kit (Applygen Technologies Inc., P1250). Proteins were separated by SDS-PAGE and then transferred to PVDF membranes. Membranes were incubated with primary antibody for $12 \mathrm{~h}$ at $4^{\circ} \mathrm{C}$ and then washed 3 times for $15 \mathrm{~min}$. Membranes were incubated with secondary antibodies for $1 \mathrm{~h}$ at $37^{\circ} \mathrm{C}$. Finally, membranes were scanned with an Odyssey Infrared Imaging System (LI-COR, Lincoln, NE).

\section{Evans Blue Staining}

Mice were intraperitoneally injected with a $4 \mu \mathrm{l} / \mathrm{g}$ dose of a $1 \%$ sterile normal saline solution of Evans Blue dye and sacrificed 24 hours later. Sciatic nerves were cut into $10-\mu \mathrm{m}$ sections and then examined under a fluorescence microscope (Olympus FV1000).

\section{Behavioral Analysis}

The animals' motor coordination and balance were assessed using a rotarod apparatus at P120 (2-80 rpm Rota-Rod 47, 600; Ugo Basile, Comerio, Italy). Mice were trained 4 times before being tested. The rotarod apparatus was accelerated from 15 to $40 \mathrm{rpm}$. The ratio was increased by $2.5 \mathrm{rpm} / \mathrm{min}$. The time each animal remained on the rotarod was recorded. All mice was tested 3 times, with a 10-min break between each test.

\section{Quantitative Real-time PCR}

Mice were sacrificed and then sciatic nerves were frozen in liquid nitrogen. Total RNA was extracted using the RNeasy Lipid Tissue Mini Kit (QIAGEN, 74804), according to the manufacturer's protocol. After reverse transcription using avian myeloblastosis virus (AMV) reverse transcriptase and random primers (Promega, A3500), quantitative real-time PCR (Agilent Technologies, Santa Clara, USA) was performed using SYBR Green (Generay Biotech Co., Ltd., GK8020). All primers are shown in Table 1.

\section{Bromodeoxyuridine (BrdU) and TUNEL Staining}

For BrdU incorporation studies, mice were intraperitoneally injected with BrdU (50 mg per $\mathrm{kg}$ bodyweight) $2 \mathrm{~h}$ before sacrifice. Sciatic nerves from the mice were cut into $8-\mu \mathrm{m}$ longitudinal
Table 1. The primer sequences used for qRT-PCR

\begin{tabular}{lcc}
\hline Gene & Forward primer & Reverse primer \\
\hline MPZ & CTGGTCCAGTGAATGGGTCT & CATGTGAAAGTGCCGTTGC \\
MBP & AATCGGCTCACAAGGGATTCA & TCCTCCCAGCTTAAAGATTTGG \\
MAG & TTTGGACGTCAAGTACCCCC & GGTACAGGCTCTTGGCAACT \\
c-JUN & TGACTGCAAAGATGGAAACG & CCAGGTTCAAGGTCATGCTC \\
EGR1 & CAGCGCCTTCAATCCTCAAG & AGCGGCCAGTATAGGTGATG \\
EGR3 & ATGGACATCGGTCTGACCAAC & AAAAGATTGCTGTCCAAGGC \\
P75 & CAACCAGACCGTGTGTGAAC & GGAGAACACGAGTCCTGAGC \\
CD68 & GGATTGGATTGAGGAAGGAACTG & GCCGCATGGCAGAGATG \\
TNF- $\alpha$ & AGCCCACGTCGTAGCAAACCAC & AGGTACAACCATCGGCTGGCA \\
TGF- $\beta$ & TGCGCTTGCAGAGATTAAAA & TGCCGTACAACTCCAGTGAC \\
MCP1 & AGGTCCCTGTCATGCTTCTG & GCTGCTGGTGATCCTCTTGT \\
CD86 & GTTACTGTGGCCCTCCTCCTT & CTGATTCGGCTTCTTGTGACATA \\
GAP-43 & TGGTGTCAAGCCGGAAGATAA & GCTGGTGCATCACCCTTCT \\
Cyclin D1 & GCGTACCCTGACACCAATCT & CACAACTTCTCGGCAGTCAA \\
GDNF & GATATTGCAGCGGTTCCTGT & AACATGCCTGGCCTACTTTG \\
BDNF & GGACTCTGGAGAGCGTGAAT & CGAACCTTCTGGTCCTCATC \\
IGF-1 & CCACACTGACATGCCCAAGACTCA & TGTTTTGCAGGTTGCTCAAGCA \\
CNTF & TGGCTAGCAAGGAAGATTCG & CATCTCACTCCAGCGATCAG \\
PTN & TTTTCATCTTGGCAGCTGTG & ACACTCCACTGCCATTCTCC \\
GAPDH & ATGACATCAAGAAGGTGGTG & CATACCAGGAAATGAGCTTG \\
\hline
\end{tabular}


sections. The section was incubated with an ani-S100 antibody for $12 \mathrm{~h}$ at $4^{\circ} \mathrm{C}$ after being permeabilized with Trition-X100 for $30 \mathrm{~min}$. After an incubation with secondary antibodies, sections were incubated with $2 \mathrm{~N} \mathrm{HCl}$ for $30 \mathrm{~min}$ at $37^{\circ} \mathrm{C}$ and then neutralized with borate buffer $(0.1 \mathrm{M}, \mathrm{pH} 7.5)$ for $12 \mathrm{~min}$. Samples was incubated with anti-BrdU antibodies and secondary antibodies before being observed under a fluorescence microscope (Olympus FV1000).

TUNEL staining was detected using a one-step TUNEL apoptosis assay kit. Longitudinal sections of the nerve were incubated with the reaction mixture for $2 \mathrm{~h}$ at $37^{\circ} \mathrm{C}$ after being permeabilized with $0.1 \%$ Triton $\mathrm{X}-100$. Nuclei were stained with Hoechst dye and were observed under a fluorescence microscope (Olympus FV1000).

\section{Immunofluorescence Staining}

Sciatic nerves were dissected and cut into 8- $\mu$ m longitudinal sections. Sections were washed 3 times with PBS for 15 min each. After the sciatic nerves sections were blocked with $0.3 \%$ Triton X-100 PBS for $30 \mathrm{~min}$ at room temperature, they were incubated with primary antibodies for $12 \mathrm{~h}$ at $4^{\circ} \mathrm{C}$. Longitudinal sections were washed 3 times for $15 \mathrm{~min}$ and then incubated with secondary antibodies for $2 \mathrm{~h}$ at $4^{\circ} \mathrm{C}$. After washes with PBS, sections were observed under a fluorescence confocal microscope (Olympus FV1000).

\section{Histology and Immunohistochemistry}

The mice were sacrificed and perfused with $4 \%$ paraformaldehyde. Sciatic nerves were cut into $8-\mu \mathrm{m}$ longitudinal sections. Sections were washed three times for 15 min and then incubated with $3 \% \mathrm{H}_{2} \mathrm{O}_{2}$ for 15 min. After longitudinal sections of the sciatic nerve were permeabilized with $0.3 \%$ Triton X-100 PBS for 30 min, they were incubated with the primary antibodies for $12 \mathrm{~h}$ at $4^{\circ} \mathrm{C}$. Sections were then incubated with a biotinylated secondary antibody for $30 \mathrm{~min}$ at $37^{\circ} \mathrm{C}$, and the longitudinal sections of the sciatic nerve were incubated with horseradish peroxidase (HRP)-conjugated streptavidin for $30 \mathrm{~min}$ at $37^{\circ} \mathrm{C}$. Sections were incubated for 2 min with a 3, 3'-diaminobenzidine (DAB) solution and then observed under the microscope (Olympus, Tokyo, Japan).

\section{Toluidine Blue Staining and Electron Microscopy}

Mice were sacrificed and the sciatic nerves were perfused with $4 \%$ glutaraldehyde in $0.1 \mathrm{M} \mathrm{PBS,} \mathrm{pH} \mathrm{7.4,}$ for $24 \mathrm{~h}$. After the sciatic nerves were washed three times, they were stained with osmium tetroxide for $1 \mathrm{~h}$, and then washed with PBS three times. After dehydration in a gradient of ethanol solutions, sciatic nerves were embedded in resin. Semi-thin sections $(0.5 \mu \mathrm{m})$ were cut, stained with toluidine blue, and observed under a microscope (Olympus, Tokyo, Japan).

Ultra-thin cross-sections (50-nm thick) were cut for the electron microscopy analysis. The ultra-thin cross-sections were stained with lead citrate and uranyl acetate before being observed under a transmission electron microscope (TEM, JEM-1230).

\section{Statistical Analysis}

Each experiment was performed using at least three mice. Values are expressed as means \pm standard errors of the means (SEM) and the data were analyzed using SPSS 21.0 software. Data from different groups were analyzed using by Student's t test, ANOVA, or the Mann-Whitney U-test, as appropriate. Statistics were considered significant when $\mathrm{P}<0.05$.

\section{Results}

\section{The Mutant Sod1 Gene Does Not Impair Myelination in the Developing Nerves}

Peripheral nerves develop from the neural crest. SCs establish a 1:1 relationship with and myelinate large caliber axons. The development of the PNS is mostly complete 4 weeks after birth, ensuring the effective and rapid transmission of signals $[8,24]$. We first examined the changes in S0D1-G93A mice in detail at different stages of peripheral nerve development to investigate deficits in the developing nerves. For this purpose, mutant mice were used to investigate the ultrastructure of the PNS and morphological changes during the development of the sciatic nerve compared to the profiles and characteristics of fibers in the sciatic nerves 
of littermate control mice.

According to the electron microscopy results, sciatic nerves from $\mathrm{P} 15$ and P30 SOD1-G93A mice showed no differences compared to littermate control nerves (Figs. 1A-D (for all online suppl. material, see www.karger.com/ doi/10.1159/000489627) and S1). The percentage of myelinated fibers in mutant mice at each age remained unchanged (Fig. 1E). Moreover, the measurement of the g-ratio (the axon diameter divided by the myelinated axon diameter) did not show significant differences between control and mutant mice at P30 (Fig. 1F).

Mpz, Mbp and Mag are myelin structural proteins that are crucial for myelination. c-Jun acts as an important transcription factor in injured nerves [25]. Based on the qRT-PCR results, levels of these mRNAs in the sciatic nerves of mutant mice were similar to control mice at P30 (Fig. 1G). Thus, the mutant human Sod 1 gene may not affect PNS development.

\section{Progressive Axonal Degeneration is} Observed in the SOD-G93A Mice

Peripheral nerves from mutant mice were examined at P60 to further examine the toxicity of the mutant SOD1 protein in the early stage of ALS. The sciatic nerves of SOD1-G93A mice did not reveal significant differences in the abnormal fibers compared with the littermate controls, consistent with behaviors observed at P60 (Fig. 2A-D).

At the age of 90 days, the SOD1-G93A mice appeared to display weakness and tremors that are characteristic of muscle atrophy and peripheral neuropathy. In our study, the number of motor neurons in the spinal cord of the mutant was distinguished from control sections, indicating that the motor deficits observed in the mutant mice may be caused by neuronal loss (see online suppl. material, Fig. S2).

Semi-thin cross-sections of sciatic nerves were analyzed by light microscopy to investigate the gross morphology of the nerve fibers in mutant mice. The mutant nerves appeared to be damaged, with more abnormal fibers than nerves of littermate control mice (Fig. 2K). Based on these data, the mutant Sod1 gene leads to the degeneration of axons and myelin sheaths at P90. Moreover, a detailed examination of nerves from mutant mice at P90 indicated that abnormal fibers were grouped together in one area (Fig. 2H),

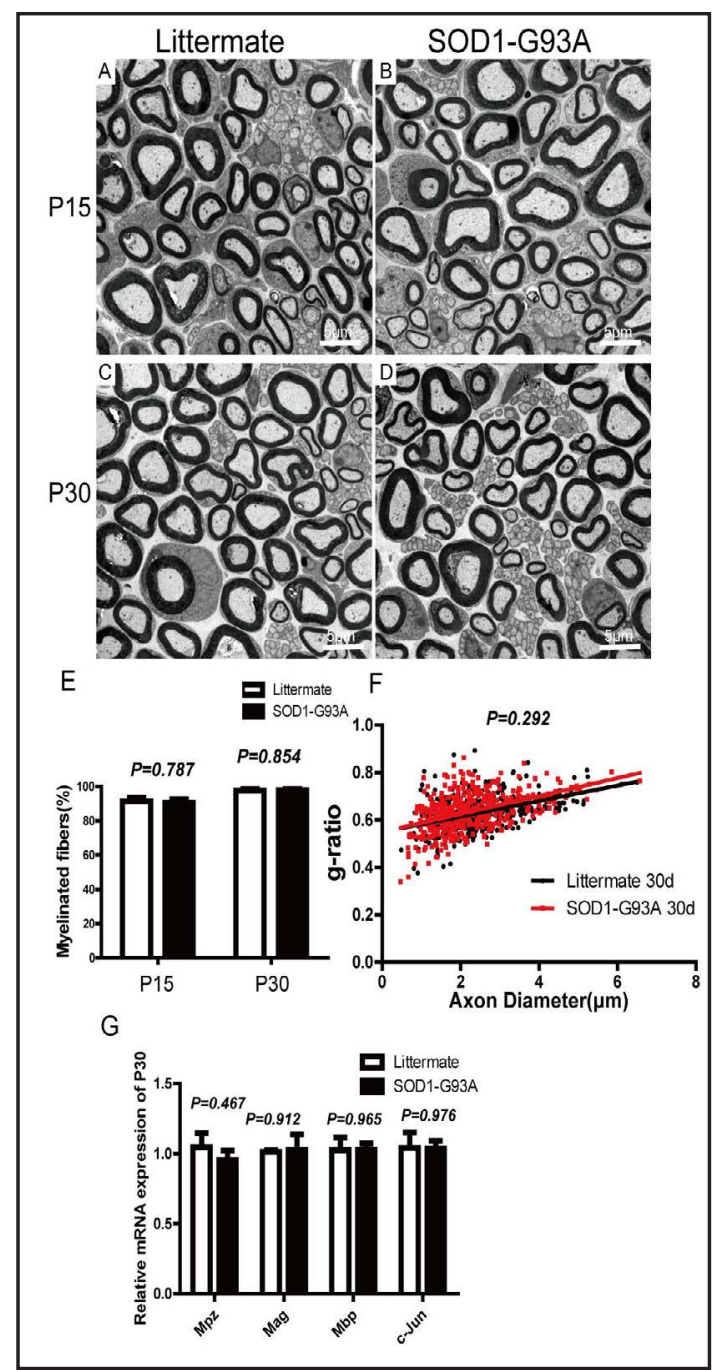

Fig. 1. SOD1-G93A mice exhibited normal myelination in the developing nerve. (A-D) Representative electron micrographs from transverse ultra-thin sections of the sciatic nerves from SOD1-G93A and littermate control mice at postnatal ages P15 and P30. No differences in the myelinated fibers were observed between the two groups. (E) Histomorphometric quantification of myelinated fibers in the SOD1-G93A and littermate control mice at P15 and P30.At P30, myelination is almost complete. (F) An analysis of the g-ratios and axon diameters shows a lack of changes in SOD1-G93A mice at P30.(G) Expression levels of the Mpz, Mag, Mbp, and c-Jun mRNAs in SOD1-G93A and littermate control mice at P30 ( $\mathrm{n}=3$ mice per group at P15 and P30; data are presented as means \pm SEM). 
Deng et al.: Progressive Degeneration and Inhibition of Peripheral Nerve Regeneration in the Amyotrophic Lateral Sclerosis Mouse Model

Fig. 2. Morphological alterations and progressive axonopathy in mutant nerves. (A-D) Morphological assessment of axonal and myelin degeneration in sciatic nerves from SOD1-G93A and littermate control mice. Toluidine blue staining of semi-thin transverse sections of sciatic nerves at P60 is shown. Representative electron micrographs from transverse ultra-thin sections of the sciatic nerves from SOD1G93A and littermate control mice at P60.(E-H) Toluidine blue staining of semi-thin transverse sections of sciatic nerves at P90. (I-J) Electron microscopy of transverse sections of SOD1-G93A and littermate control mice at P90. Arrows indicate normal fibers, and arrowheads indicate abnormal fibers.(K) The percentage of abnormal fibers in the SOD1G93A and littermate control mice was quantitated, and the difference between the two groups at P90 was significant $(\mathrm{n}=3$ mice per group at P60 and P90; data are presented as means \pm SEM).

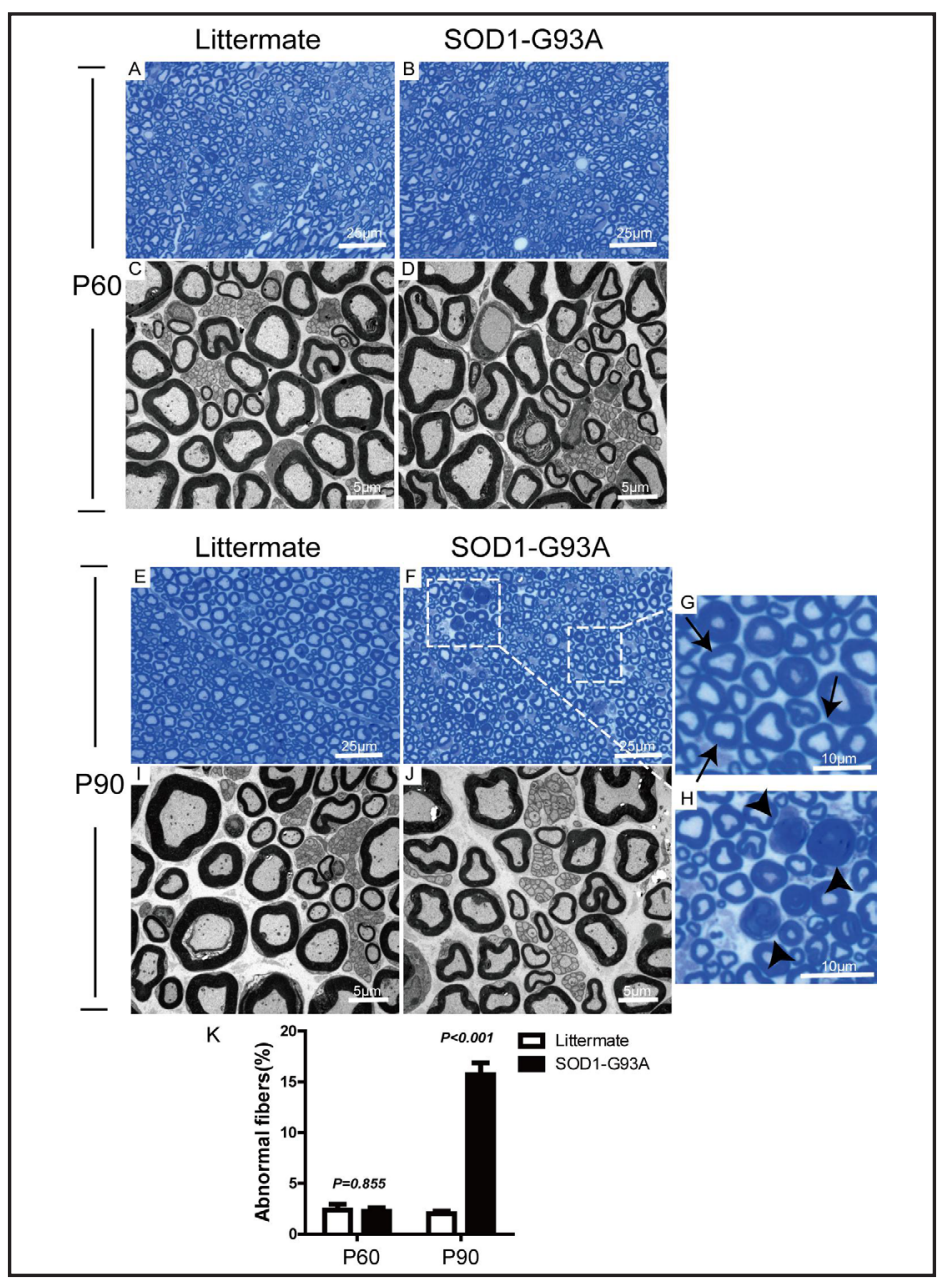

whereas normal fibers were grouped together in another area (Fig. 2G). The regional nerve damage observed in mutant mice may be a reason for the selective loss of neurons in the CNS.

\section{Severe Nerve Injury and Motor Impairments in Mutant Mice}

The SOD1-G93A mice appeared abnormal approximately 90 days after birth. The mutant mice exhibited a 38\% decrease in body weight (data not shown) and were completely paralyzed, exhibiting muscle atrophy and dragging their hind legs when they moved (see online suppl. material, Fig. S3B) at P120. Rotarod behavioral tests were performed to analyze the neuromuscular deficit at P120. The mutant mice and littermate controls were placed on a rotating rod and the time they remained on the apparatus was measured. The mutant mice exhibited a substantial locomotor deficit compared with the control group (see online suppl. material, Fig. S3A).

We further investigated the morphology of the sciatic nerves from the mutant and littermate control mice at P120 using transverse semi-thin sections and electron microscopy. An examination of the axons and myelin sheaths showed large numbers of abnormal fibers in the SOD1-G93A mice (Fig. 3C and D). Moreover, nerve abnormalities included degeneration of myelinated axons that were filled with myelin debris and disintegration of nonmyelinated axons (Fig. 3F-I).

Furthermore, qRT-PCR data showed that c-Jun mRNA levels were substantially increased in mutant nerves compared with those in control nerves (Fig. 3J). These data were consistent with Western blots showing that neurofilament $160 \mathrm{kDa}$ (NF-160) expression was downregulated during axonal degeneration (Fig. 3K).

\section{KARGER}




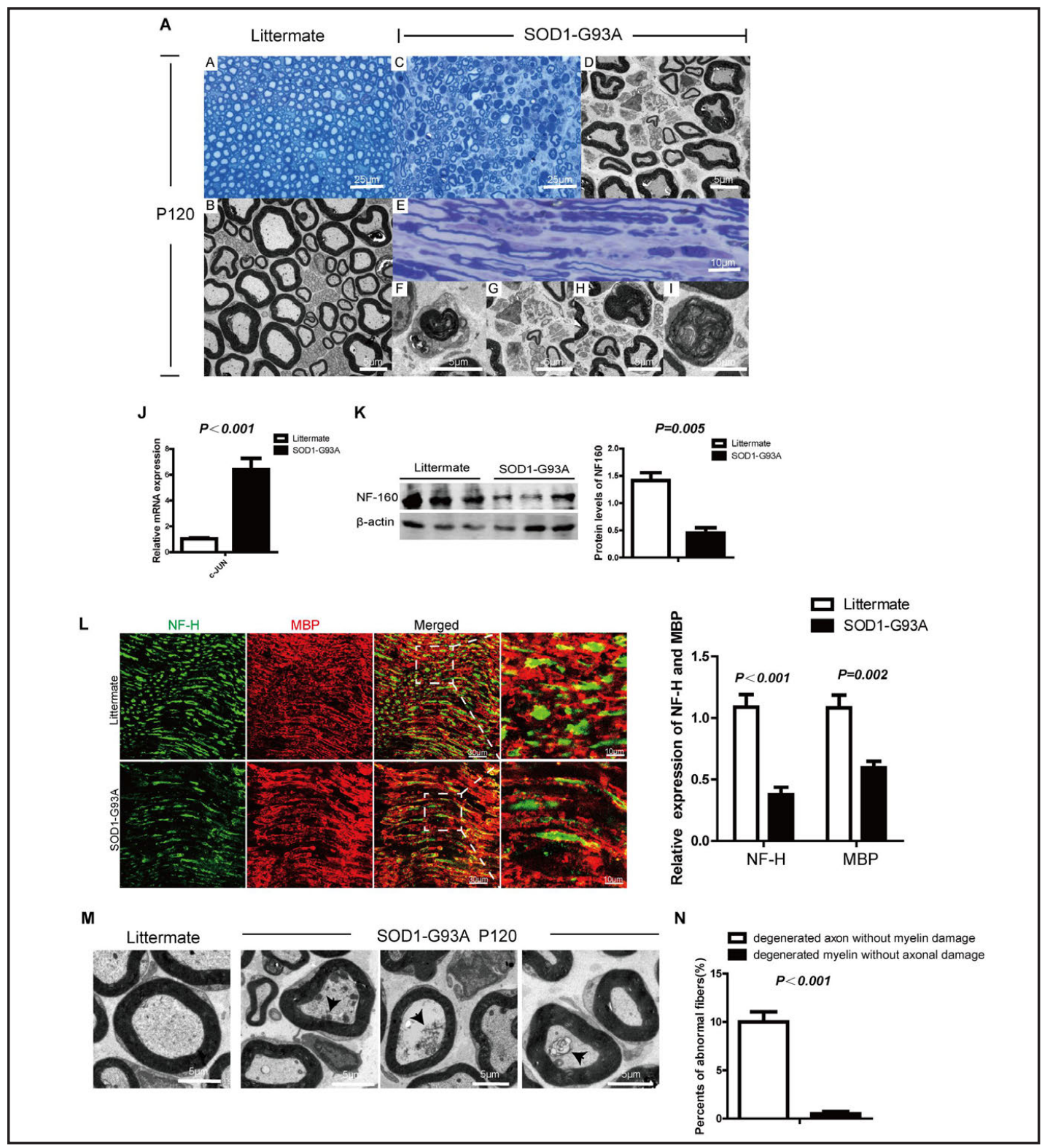

Fig. 3. Axon degeneration in SOD1-G93A mutants. (A-D) Semi-thin transverse sections and ultra-thin transverse section of sciatic nerves from SOD1-G93A and littermate control mice at P120. (E) Toluidine blue staining of semi-thin longitudinal sections of sciatic nerves show severe degeneration of axons and myelin in mutant mice. (F-I)Abnormal fibers in the SOD1-G93A mice. (J) A PCR analysis showed that the c-Jun mRNA level was substantially decreased in the SOD1-G93A sciatic nerve at P120. (K) Western blots of the NF-160 protein in littermate control and SOD1-G93A sciatic nerves. Levels of the NF-160 protein were decreased in mutant nerves at P120. (L)Double immunofluorescence staining of NF-H (a neurofilament marker) and MBP (a myelin marker) was performed in the peripheral nerve of SOD1-G93A and littermate control mice at P120.The expression of NF-H and MBP in the SOD1-G93A and littermate control mice was quantitated, and the difference between the two groups showed that axons and myelin were damaged severely at P120 ( $\mathrm{n}=$ 3 mice per group at P120; data are presented as the means \pm SEM).(M)Electron microscopy of transverse sections of SOD1-G93A (degeneration of axons without myelin damage) and littermate control (normal fibers) mice at P120.(N)The percentage of abnormal fibers (including fibers with degenerated axons without myelin damage, fibers with degenerated myelin without axonal damage) in the S0D1-G93A mice at P120 was calculated, and the difference between the two groups show that axonal degeneration may occur earlier than degeneration of myelin in the mutant mice.

\section{KARGER}


Due to the severe toxicity of mutant SOD1, the peripheral nerves are progressively denatured, which is mainly reflected in the degeneration of Schwann cells and axons. At P120, the sciatic nerves of mutant mice were examined in detail, and we found that most axons and myelin sheaths had been damaged (Fig. 3F,H,I). However, a few of the myelin sheaths retained a healthy morphology despite abnormalities in the axons (Fig. 3L).

Statistical analysis was performed, and we found that the number of degenerated myelinated axons (degeneration of axon) was significantly higher than the number of degenerated myelinated axons with myelin damage (degeneration of both axon and myelin) (Fig. 3L). These results suggest that axonal degeneration may occur earlier than degeneration of myelin or Schwann cells.

In addition, immunofluorescence analysis of the axon and myelin sheath was performed to investigate the relationship between the axon and the myelin sheath in the SOD1-G93A mice. The results showed that the expression of NF-H (an axonal marker) decreased significantly, while MBP (a marker for myelin sheaths) showed a smaller decrease than that for NF-H (Fig. 3M). These results may indicate early axonal damage, prior to damage to myelin or to Schwann cells and myelin, and degeneration of the Schwann cell and myelin may be a secondary effect caused by axon degeneration.

It has been reported that conditional knockout of a mutated SOD1 gene in Schwann cells did not affect the numbers and distributions of axonal diameters in L5 motor roots [26], which may be consistent with the results we achieved.

\section{Formation of a Pro-inflammatory Environment in Mutant Nerves}

After peripheral nerve injury, the pathology involves axonal degeneration and the breakdown of the myelin sheath. Inflammatory cells have been shown to contribute to the progression of nerve injury [27]. The inflammatory response, a mechanism by which many inflammatory cells remove necrotic cellular debris, plays a crucial role in nerve injury. Macrophages are recruited and then release molecules in response to peripheral nerve injury, including interleukin-1, neurotrophic factors and transforming growth factor (TGF) $[19,28]$. In our study, the expression of neurotrophic factors in mutant nerve was examined. Levels of insulin-like growth factor 1 (IGF-1), glial-derived neurotrophic factor (GDNF) and pleiotrophin (PTN) were increased, whereas levels of ciliary neurotrophic factor (CNTF) were decreased (see online suppl. material, Fig. 4S).

The expression of CD68, a common marker of activated macrophages, was significantly increased during the inflammatory response. Monocyte chemoattractant protein-1 (MCP1 ) is a chemokine that is secreted in response to inflammation. The quantification of inflammatory factors revealed that levels of the Cd68, Mcp-1, Cd86 and Tgf- $\beta$ mRNAs were increased, whereas mRNA levels of the pro-inflammatory cytokine tumor necrosis factor- $\alpha$ (Tnf- $\alpha$ ) remained unchanged in sciatic nerves from P120 mutant mice (Fig. 4A).

Furthermore, the expression patterns of CD68, MCP-1, CD86 and TGF- $\beta$ were detected by immunofluorescence staining and we observed the same differences between the two groups (Fig. 4B-F). A large number of activated macrophages accumulated during peripheral nerve injury in mutant mice.

\section{SC Survival is Impaired and the Blood-Nerve Barrier (BNB) Exhibits Deficits}

The degeneration of axons and myelin in the PNS was so severe that we considered whether SC survival was affected at P120. For these purposes, investigated apoptosis in nerves from mutant and littermate control mice. TUNEL staining was performed to detect SC apoptosis in the two groups (Fig. 5A). A significant increase in apoptosis was observed in the mutant nerves (Fig. 4B) compared to the littermate control mice. The results were consistent with the morphology and behaviors we have observed.

The BNB plays an important role in the degeneration of axons and myelin after peripheral nerve injury. Furthermore, BNB dysfunction after nerve injury is regulated by macrophages. Sciatic nerve injury correlates with the breakdown of the BNB and the accumulation of macrophages [29]. Peripheral nerve injury is a complicated process of axonal degeneration,

\section{KARGER}


Deng et al.: Progressive Degeneration and Inhibition of Peripheral Nerve Regeneration in the Amyotrophic Lateral Sclerosis Mouse Model

Fig. 4. Mutant nerves exhibit a strong inflammatory response. (A) qRT-PCR analysis of mRNA levels of inflammatory factors in SOD1-G93A and littermate control mice at P120.(B-E) Immunofluorescence staining of inflammatory factors (CD68, MCP1, CD86 and TGF- $\beta$ ) and NF-H on the peripheral nerve of the two groups.(F)Levels of the inflammatory factors CD68, MCP1, CD86 and TGF- $\beta$ were increased in the mutant nerve at $\mathrm{P} 120$ ( $\mathrm{n}=3$ mice per group at P120; data are presented as means \pm SEM).

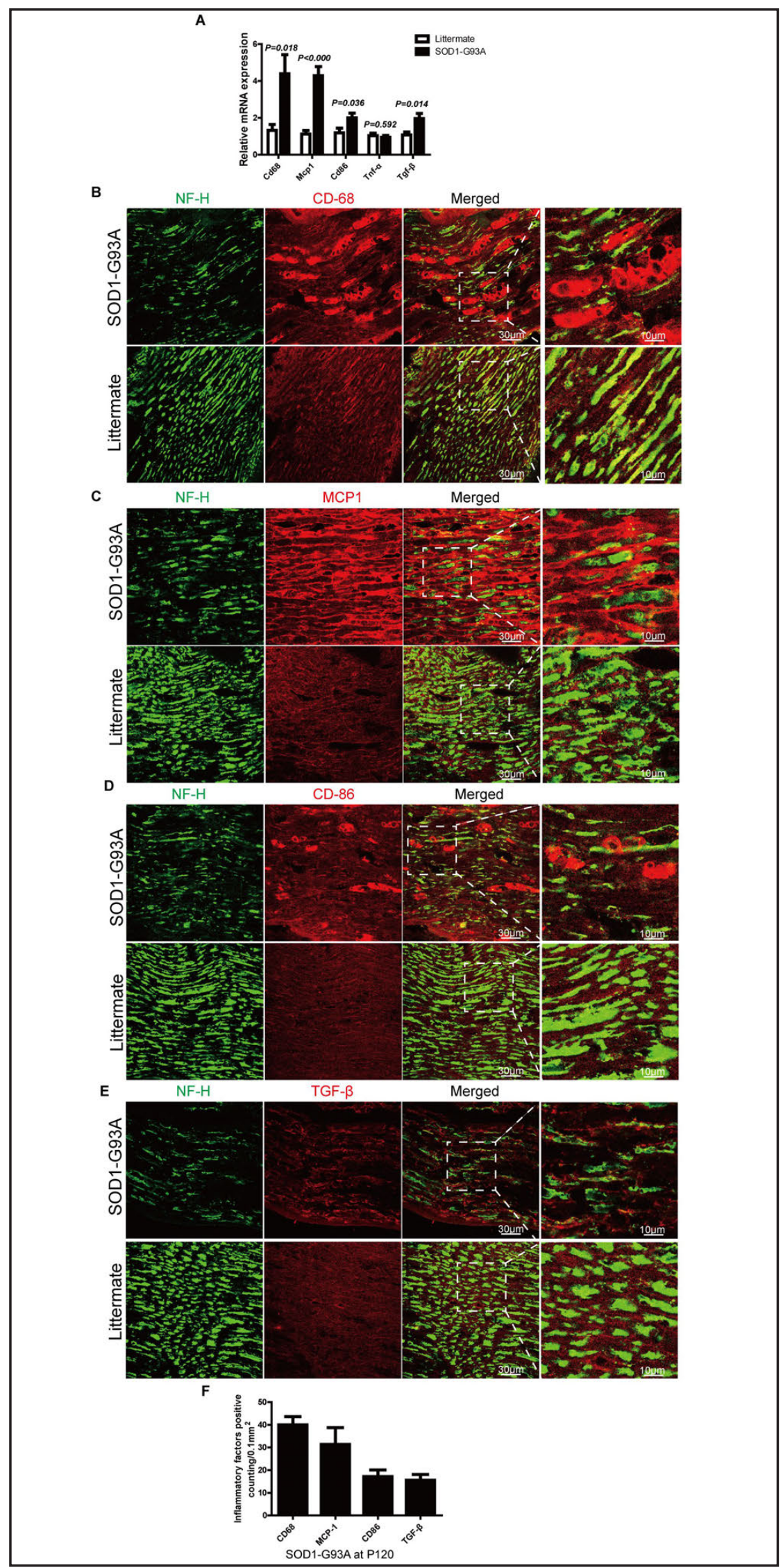


myelin breakdown and BNB compromise. A breach in the BNB is associated with axon degeneration after peripheral nerve injury. Mice were injected with Evans blue dye, which is transported from blood vessels to the endoneurium in the presence of a BNB defect, $24 \mathrm{~h}$ before sacrifice to explore the effects of the SOD1 mutation on the BNB. Evans Blue leakage indicated that the sciatic nerve was severely damaged in S0D1-G93A mice at P90 and P120 (Fig. 5C and D).

Inhibition of Macrophage Recruitment and SC Proliferation in Mutant Nerve after Crush Injury

Wallerian degeneration involves axon and SC degeneration, macrophage recruitment, the removal of cell debris and the regeneration of the PNS[19]. SCs differentiate into myelinating or nonmyelinating SCs during PNS development [8]. SCs are plastic in that they have the ability to convert back to a precursor form upon nerve damage, which allows the PNS to recover from injury. Axons are guided to regrow by Bungner cells, and macrophages are recruited to clear the myelin debris [30,31]. The mechanisms that govern PNS regeneration are not fully understood.

PNS regeneration relies on axons, SCs and immune cells. Macrophages are recruited and accumulate, contributing to degeneration and axonal regeneration [31]. In our study, we performed a sciatic nerve crush injury in P30 SOD1-G93A and littermate control mice. Based on the level of the CD68 mRNA, a greater number of macrophages were recruited in the nerves from littermate control mice 7 days after crush (Fig. 6C) compared to the mutant nerves. Further detection of CD68 using immunostaining indicated that an increased number of macrophages accumulated at the crush site in the littermate control mice 7 days after crush (Fig. 6D and E). Thus, SOD1-G93A inhibits the recruitment of inflammatory cells.

Levels of the p75 neurotrophin receptor (p75NTR), Egr-1 and Egr-3, which are expressed by immature SCs, were increased after transection. Mutant nerves from SOD1-G93A mice displayed a 57\% reduction in Egr-1 mRNA levels and a 19\% reduction in Egr-3 mRNA levels

Fig. 5. SC death is increased and BNB defects are observed. (AB) TUNEL staining in longitudinal sections of SOD1-G93A and littermate control mice; nuclei were counterstained with Hoechst dye. The number of TUNEL-positive nuclei increased in mutant mice at P120.(C-D) Fluorescent images of Evans Blue staining in SOD1-G93A and littermate control sciatic nerves at P30, P60, P90 and P120. The Evans Blue staining showed that the sciatic nerve was progressively destroyed(n = 3 mice per group; data are presented as means \pm SEM).

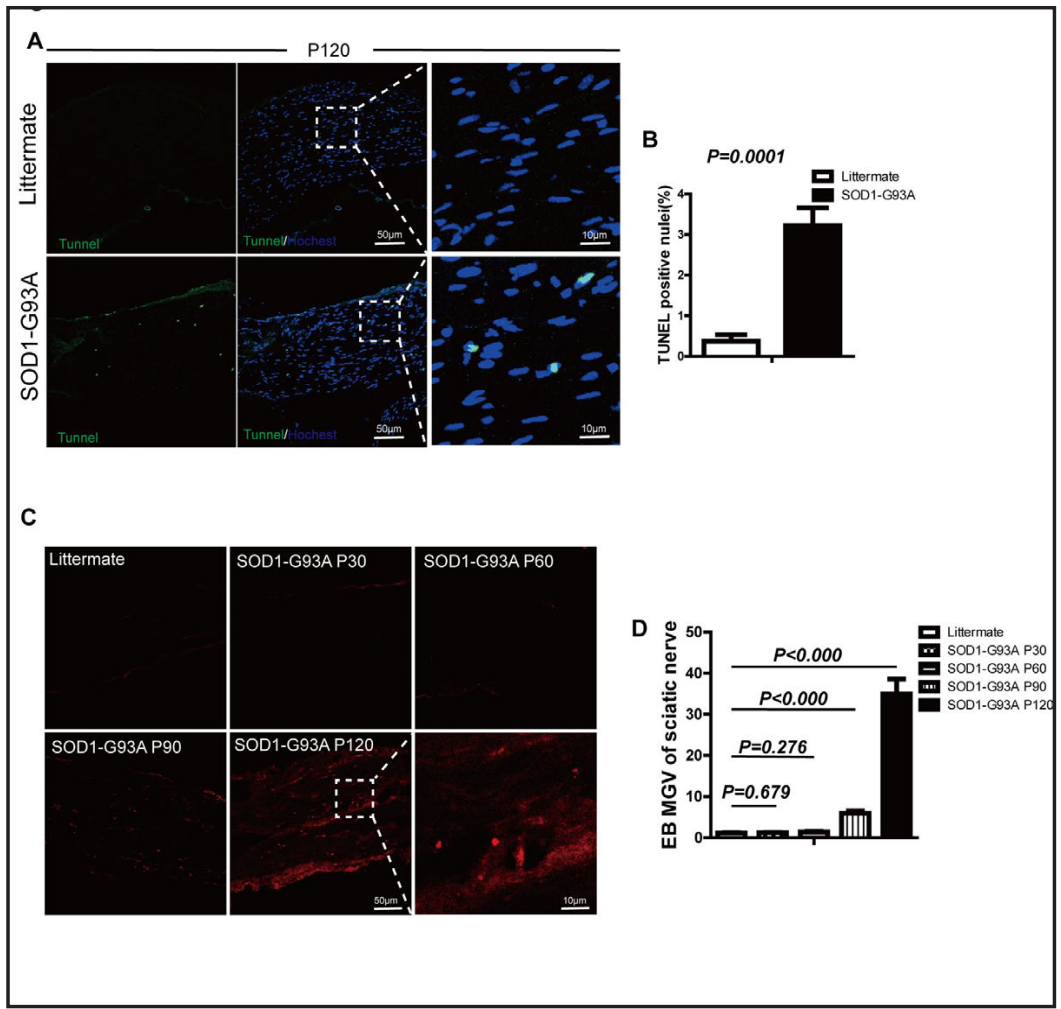


Deng et al.: Progressive Degeneration and Inhibition of Peripheral Nerve Regeneration in the Amyotrophic Lateral Sclerosis Mouse Model

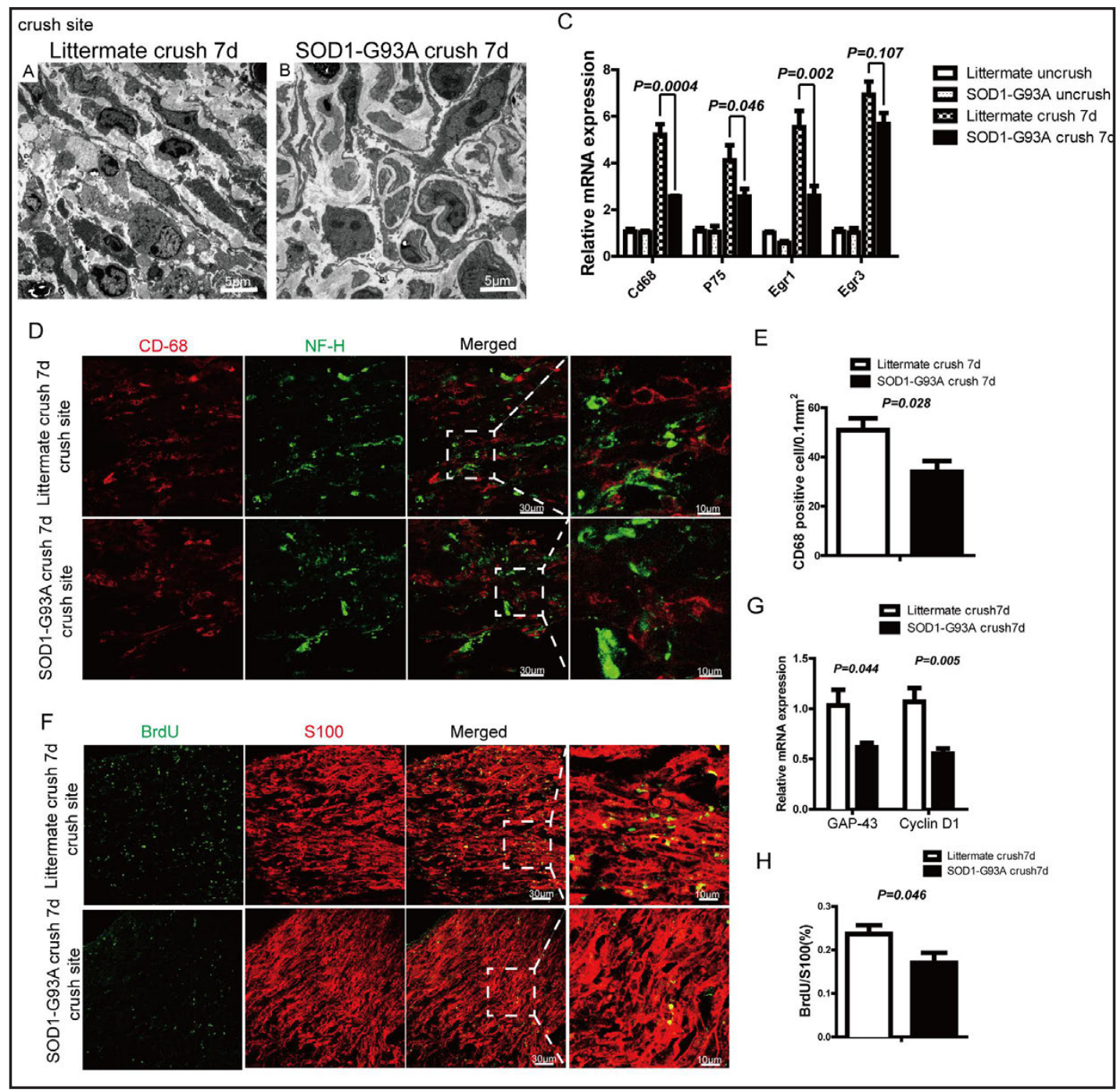

Fig. 6. The mutant Sod1 gene affects the inflammatory response and cell proliferation in crushed nerves. (A-B) Representative electron micrographs from SOD1-G93A and littermate control mice obtained 7 days after crush injury (nerves were crushed at P30).(C) Levels of the Cd68, p75, Egr-1 and Egr-3 mRNAs were decreased in SOD1-G93A mice 7 days after crush injury compared with those seen in littermate control mice (the uncrushed and crushed mice were the same age).(D-E) Images of immunofluorescence staining of mutant and control sciatic nerves 7 days after crush at the crush site. A greater number of macrophages was recruited and accumulated in the littermate control mice. ( $\mathrm{F}$ and $\mathrm{H}$ ) BrdU staining of mutant and control nerves 7 days after crush; less cell proliferation was observed in SOD1-G93A nerves.(G) qRT-PCR analysis of Gap43 and Cyclin D1 mRNA levels in mutant and control nerves 7 days after crush ( $\mathrm{n}=3$ mice per group; data are presented as means \pm SEM).

by qRT-PCR, whereas the level of the p75Ntr mRNA exhibited a 39\% decrease in the mutant nerve 7 days after crush (Fig. 6C). The mutant Sod1 gene significantly affected SC plasticity.

Proliferation was analyzed using BrdU staining to investigate whether mutant SOD1 affected cell proliferation after crush injury (Fig. 6F). Similar to S100-positive cells, a greater number of BrdU-positive cells was observed in the nerves from littermate control mice 7 days after crush (Fig. 6H). This result was consistent with the levels of the growth-associated protein 43 (Gap43) and Cyclin D1 mRNAs (Fig. 6G). 
Fig. 7. Axonal regeneration is abnormal in SOD1 mutants. (A) Electron micrographs from SOD1-G93A and littermate control mice obtained 28 days after crush injury (nerves were crushed at P30).(B) An analysis of SOD1-G93A and littermate control mice 28 days after crush injury showed that a greater number of myelin fibers was detected in the littermate control mice 28 days after crush injury (the uncrushed and crushed mice were the same age).(C) The diameters of the myelin fibers in the two groups were measured, and myelin fibers displayed larger diameters in littermate control mice 28 days after crush injury ( $n=3$ mice for each group; data are presented as means \pm SEM).

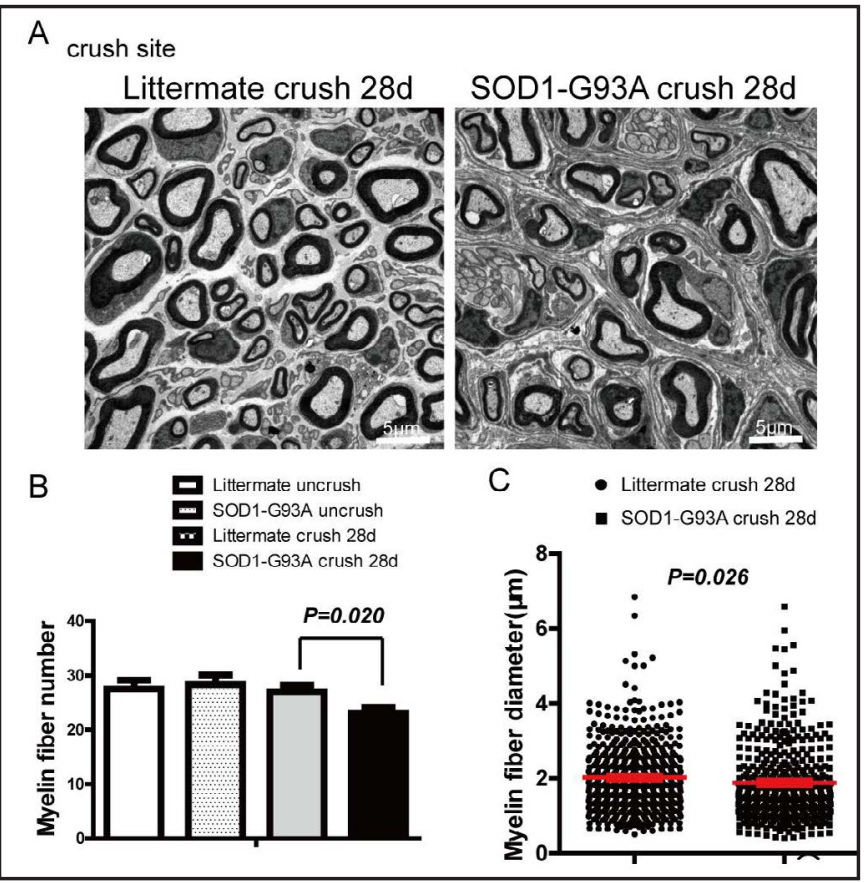

Remyelination and Regeneration Defects in Mutant Mice after Crush Injury

Axonal remyelination and regeneration are crucial for the recovery of motor function after peripheral nerve injury. A nerve crush was performed at P30. Transmission electron microscopy images of the injury site were analyzed after 28 days of regeneration (Fig. 7A).

Nerves from both the SOD1 mutant and littermate control mice were remyelinated 28 days after crush injury. However, the littermate control mice exhibited more effective remyelination, as more myelinated fibers were detected in the control nerves 28 days after crush injury (Fig. 7B). Moreover, the hypomyelination in mutant nerves was primarily observed in the large caliber axons, when the diameters of the myelinated axons of the two groups were measured (Fig. 7C). In summary, we observed obvious alterations in the mutant and control mice 28 days after crush injury.

\section{Discussion}

In this study, disease progression leads to the degeneration of axons and myelin in the PNS of older SOD1 mutant mice. Macrophages were recruited and accumulated, and they were associated with the BNB deficit during peripheral nerve injury. Moreover, regeneration and remyelination were detected in SOD1-G93A mice at an early time point.

Peripheral nerves develop from the neural crest, and the neural crest gives rise to Schwann cell precursors (SCPs)[8]. SCPs are a source of SCs and a small population of fibroblasts in the PNS. After birth, SCs establish a 1:1 relationship with large caliber axons to ensure the integrity and normal function of the PNS[8, 24]. SCs and axons did not show differences during the development of mutant mice. However, axons and SCs degenerated at a later stage in the mutant mice.

Mutant SOD1 is implicated in a toxic gain of function phenotype [32]. During the progression of disease, peripheral nerve dysfunction occurs. Based on the changes in the PNS observed in mutant mice, the time at which axons and myelin underwent rapid injury occurred after P90, which was usually regarded as the time of onset. Thus, the PNS and BNB were largely damaged due to the toxicity of the mutant SOD1 protein. According to a previous large-scale gene profiling study, oxidative stress and mitochondrial dysfunction occur in the 
sciatic nerves of model mice, which were linked to the degeneration and deterioration of the axons and myelin [33-35]. At 120 days, defects in axons and myelin were more severe and the mutant mice appears to have difficulty walking, as motor function was limited.

The inflammatory response to nerve injury comprises macrophage infiltration and the subsequent engulfment and removal of myelin debris $[19,36]$. MCP-1, a monocyte chemoattractant that functions after acute peripheral nerve injury, is released from the injured nerve, and then numerous immune cells are recruited to the injured nerve, where they establish a pro-inflammatory environment and play a role in phagocytosis, thus contributing to the microenvironment of the injured nerve [37, 38]. A large number of macrophages were detected at the injury site, and levels of inflammatory factors, such as CD68 and CD86, were increased. Tnf- $\alpha$ expression remained unchanged, suggesting that some inflammatory factors were not activated during peripheral nerve injury.

When axons were crushed in the PNS of SOD1-G93A mice, the axons and myelin progressively degenerated and the distal portion was degraded; the process was accompanied by the recruitment of a large number macrophages $[9,39]$. The immune response in the SOD1G93A mouse spinal cord may be considered a contributor to motor neuron loss. Although inflammation is mainly linked to axon degeneration, the identification of the molecular mechanisms and the immune responses would promote a better understanding of the PNS. Macrophages were recently shown to modulate the activities of SCs and remove cell debris after crush injury; macrophages also promote the regeneration of axons and remyelination of SCs [19]. Seven days after crush, mutant mice accumulated fewer macrophages, suggesting an effect on the PNS regeneration.

Wallerian degeneration triggers a set of changes in peripheral nerves, including axon degeneration, recruitment of blood-borne macrophages, SC proliferation and peripheral nerve regeneration. After crush injury, SCs are responsible for the breakdown and degeneration of myelin sheaths. Dedifferentiation of mature SCs is an important feature of peripheral nerve injury [39]. A reversal of gene and protein expression occurs in which the characteristics of mature SCs are reverted to immature SCs [9]. Levels of p75NTR, a protein marker of immature SCs, are increased in the injured nerve. The level of the p75NTR mRNA suggests that SOD1-G93A mice are less equipped to prepare for regeneration and remyelination, which would contribute to recovery and affect the plasticity of peripheral nerves.

The progression of ALS in SOD1-G93A mice accelerated after crush injury due to the possible increase in metabolic demand [40]. This repair capacity of the PNS after crush injury includes SC hyperproliferation, remyelination and regeneration, followed by functional recovery after nerve injury [31]. Thus, SOD1-G93A mice displayed defects in proliferation after crush injury, leading to fewer immature SCs that give rise to mature SCs, which is particularly important for the remyelination of injured axons. The number of myelinate axons in the SOD1-G93A mice decreased 28 days after crush injury, and large-diameter axons were not well myelinated. Therefore, the toxicity of the mutant SOD1 protein may have affected the macrophage recruitment, SC proliferation and axon growth, resulting in less regeneration and remyelination of axons in the injured peripheral nerve.

In conclusion, SOD1-G93A mice exhibit a toxic gain of function phenotype, and mutant mice suffer from severe alterations to peripheral nerves. These mice develop an age-related peripheral neuropathy, in which macrophages are recruited and accumulate. Mutant nerves were also deficient in axonal remyelination and regeneration after crush injury.

\section{Acknowledgements}

The authors would like to thank the National Natural Science Foundation of China (30371089 and 81171210) for providing financial support. 


\section{Cellular Physiology Cell Physiol Biochem 2018;46:2358-2372 \begin{tabular}{l|l|l}
\hline DOI: 10.1159/000489627 & $\begin{array}{l}\text { () 2018 The Author(s). Published by S. Karger AG, Basel } \\
\text { www.karger.com/cpb }\end{array}$
\end{tabular} and Biochemistry}

Deng et al.: Progressive Degeneration and Inhibition of Peripheral Nerve Regeneration in the Amyotrophic Lateral Sclerosis Mouse Model

\section{Disclosure Statement}

No conflict of interest exists.

\section{References}

1 Bruijn, LI, Miller TM, Cleveland DW: Unraveling the mechanisms involved in motor neuron degeneration in ALS. Annu Rev Neurosci 2004; 27, 723-49.

-2 Rosen DR: Mutations in cu/zn superoxide dismutase gene are associated with familial amyotrophic lateral sclerosis. Nature 1993;364:362.

3 Mancuso R, Olivan S, Mancera P, Pasten-Zamorano A, Manzano R, Casas C, Osta R, Navarro X: Effect of genetic background on onset and disease progression in the sod1-g93a model of amyotrophic lateral sclerosis. Amyotroph Lateral Scler 2012;13:302-310.

4 Barber SC, Shaw PJ: Oxidative stress in als: Key role in motor neuron injury and therapeutic target. Free Radic Biol Med 2010;48:629-641.

5 Okado-Matsumoto A, Fridovich I: Amyotrophic lateral sclerosis: A proposed mechanism. Proc Natl Acad Sci U S A 2002;99:9010-9014.

6 Alexianu ME, Kozovska M, Appel SH: Immune reactivity in a mouse model of familial als correlates with disease progression. Neurology 2001;57:1282-1289.

7 An T, Shi P, Duan W, Zhang S, Yuan P, Li Z, Wu D, Xu Z, Li C, Guo Y: Oxidative stress and autophagic alteration in brainstem of sod1-g93a mouse model of als. Mol Neurobiol 2014;49:1435-1448.

-8 Jessen KR, Mirsky R: The origin and development of glial cells in peripheral nerves. Nat Rev Neurosci 2005;6:671-682.

-9 Jessen KR, Mirsky R: Negative regulation of myelination: Relevance for development, injury, and demyelinating disease. Glia 2008;56:1552-1565.

10 Le N, Nagarajan R, Wang JY, Araki T, Schmidt RE, Milbrandt J: Analysis of congenital hypomyelinating egr2lo/lo nerves identifies sox 2 as an inhibitor of schwann cell differentiation and myelination. Proc Natl Acad Sci U S A 2005;102:2596-2601.

11 Yun B, Anderegg A, Menichella D, Wrabetz L, Feltri ML, Awatramani R: Microrna-deficient schwann cells display congenital hypomyelination. J Neurosci 2010;30:7722-7728.

12 Yu WM, Yu H, Chen ZL, Strickland S: Disruption of laminin in the peripheral nervous system impedes nonmyelinating schwann cell development and impairs nociceptive sensory function. Glia 2009;57:850859.

13 Heller BA, Ghidinelli M, Voelkl J, Einheber S, Smith R, Grund E, Morahan G, Chandler D, Kalaydjieva L, Giancotti F, King RH, Fejes-Toth AN, Fejes-Toth G, Feltri ML, Lang F, Salzer JL: Functionally distinct pi 3-kinase pathways regulate myelination in the peripheral nervous system. J Cell Biol 2014;204:1219-1236.

14 Theisen CC, Sachdeva R, Austin S, Kulich D, Kranz V, Houle JD: Exercise and Peripheral Nerve Grafts as a Strategy To Promote Regeneration after Acute or Chronic Spinal Cord Injury. J Neurotrauma 2017;34:19091914.

15 Hu P, McLachlan EM: Selective reactions of cutaneous and muscle afferent neurons to peripheral nerve transection in rats. J Neurosci 2003;23:10559-67.

-16 Alves CJ, de Santana LP, dos Santos AJ, de Oliveira GP, Duobles T, Scorisa JM, Martins RS, Maximino JR, Chadi G: Early motor and electrophysiological changes in transgenic mouse model of amyotrophic lateral sclerosis and gender differences on clinical outcome. Brain Res 2011;1394:90-104.

17 Morrison BM, Shu IW, Wilcox AL, Gordon JW, Morrison JH: Early and selective pathology of light chain neurofilament in the spinal cord and sciatic nerve of g86r mutant superoxide dismutase transgenic mice. Exp Neurol 2000;165:207-220.

18 Sharp PS, Dick JR, Greensmith L: The effect of peripheral nerve injury on disease progression in the sod1(g93a) mouse model of amyotrophic lateral sclerosis. Neuroscience 2005;130:897-910.

19 Chen P, Piao X, Bonaldo P: Role of macrophages in wallerian degeneration and axonal regeneration after peripheral nerve injury. Acta Neuropathol 2015;130:605-618.

20 Brosius Lutz A, Barres BA: Contrasting the glial response to axon injury in the central and peripheral nervous systems. Dev Cell 2014;28:7-17. 
Deng et al.: Progressive Degeneration and Inhibition of Peripheral Nerve Regeneration in the Amyotrophic Lateral Sclerosis Mouse Model

-21 Martini R, Klein D, Groh J: Similarities between inherited demyelinating neuropathies and wallerian degeneration: An old repair program may cause myelin and axon perturbation under nonlesion conditions. Am J Pathol 2013;183:655-660.

22 Jung J, Cai W, Lee HK, Pellegatta M, Shin YK, Jang SY, Suh DJ, Wrabetz L, Feltri ML, Park HT: Actin polymerization is essential for myelin sheath fragmentation during wallerian degeneration. J Neurosci 2011;31:2009-2015.

-23 Zhang L, Johnson D, Johnson JA: Deletion of nrf2 impairs functional recovery, reduces clearance of myelin debris and decreases axonal remyelination after peripheral nerve injury. Neurobiol Dis 2013;54:329-338.

-24 Mirsky R, Woodhoo A, Parkinson DB, Arthur-Farraj P, Bhaskaran A, Jessen KR: Novel signals controlling embryonic schwann cell development, myelination and dedifferentiation. J Peripher Nerv Syst 2008;13:122-135.

-25 Arthur-Farraj Peter J, Latouche M, Wilton Daniel K, Quintes S, Chabrol E, Banerjee A, Woodhoo A, Jenkins B, Rahman M, Turmaine M, Wicher Grzegorz K, Mitter R, Greensmith L, Behrens A, Raivich G, Mirsky R, Jessen Kristján R: C-jun reprograms schwann cells of injured nerves to generate a repair cell essential for regeneration. Neuron 2012;75:633-647.

26 Lobsiger CS, Boillee S, McAlonis-Downes M, Khan AM, Feltri ML, Yamanaka K, Cleveland DW: Schwann cells expressing dismutase active mutant sod1 unexpectedly slow disease progression in als mice. Proc Natl Acad Sci U S A 2009;106:4465-4470.

-27 Mueller M, Wacker K, Ringelstein EB, Hickey WF, Imai Y, Kiefer R: Rapid response of identified resident endoneurial macrophages to nerve injury. Am J Pathol 2001;159:2187-2197.

28 Barrette B, Calvo E, Vallieres N, Lacroix S: Transcriptional profiling of the injured sciatic nerve of mice carrying the wld(s) mutant gene: Identification of genes involved in neuroprotection, neuroinflammation, and nerve regeneration. Brain Behav Immun 2010;24:1254-1267.

-29 Gray M, Palispis W, Popovich PG, van Rooijen N, Gupta R: Macrophage depletion alters the blood-nerve barrier without affecting schwann cell function after neural injury. J Neurosci Res 2007;85:766-777.

30 Brown MC, Lunn ER, Perry VH: Consequences of slow wallerian degeneration for regenerating motor and sensory axons. J Neurobiol 1992;23:521-536.

31 Gordon T: Nerve regeneration: Understanding biology and its influence on return of function after nerve transfers. Hand Clin 2016;32:103-117.

-32 Orrell RW: Amyotrophic lateral sclerosis: Copper/zinc superoxide dismutase (sod1) gene mutations. Neuromuscul Disord 2000;10:63-68.

-33 Stankovic RK: Atrophy of large myelinated axons in metallothionein-i, ii knockout mice. Cell Mol Neurobiol 2005;25:943-953.

-34 Freeman OJ, Unwin RD, Dowsey AW, Begley P, Ali S, Hollywood KA, Rustogi N, Petersen RS, Dunn WB, Cooper GJ, Gardiner NJ: Metabolic dysfunction is restricted to the sciatic nerve in experimental diabetic neuropathy. Diabetes 2016;65:228-238.

-35 Sousa MM, Saraiva MJ: Neurodegeneration in familial amyloid polyneuropathy: From pathology to molecular signaling. Prog Neurobiol 2003;71:385-400.

-36 Klein D, Martini R: Myelin and macrophages in the pns: An intimate relationship in trauma and disease. Brain Res 2016;1641:130-138.

37 Perrin FE, Lacroix S, Aviles-Trigueros M, David S: Involvement of monocyte chemoattractant protein-1, macrophage inflammatory protein-1alpha and interleukin-1beta in wallerian degeneration. Brain 2005;128:854-866.

-38 Langert KA, Von Zee CL, Stubbs EB, Jr: Tumour necrosis factor alpha enhances ccl2 and icam-1 expression in peripheral nerve microvascular endoneurial endothelial cells. ASN neuro 2013;5:e00104.

-39 Glenn TD, Talbot WS: Signals regulating myelination in peripheral nerves and the schwann cell response to injury. Curr Opin Neurobiol 2013;23:1041-1048.

40 Dupuis L, Oudart H, Rene F, Gonzalez de Aguilar JL, Loeffler JP: Evidence for defective energy homeostasis in amyotrophic lateral sclerosis: Benefit of a high-energy diet in a transgenic mouse model. Proc Natl Acad Sci U S A 2004;101:11159-11164. 